\title{
Erratum: Radial acceleration relation from symmetron fifth forces [Phys. Rev. D 95, 064050 (2017)]
}

Clare Burrage, Edmund J. Copeland, and Peter Millington

(Received 17 May 2017; published 13 June 2017)

DOI: 10.1103/PhysRevD.95.129902

We correct the discussion appearing in Sec. IV of the article: In order to indicate stability of the galactic disk, we require the ratio $t \equiv T /|W| \lesssim 0.1376$ [1], where $T$ is the total rotational kinetic energy of the baryons [as given by Eq. (16a)] and $W$ is their total potential energy. The latter receives a contribution from the Newtonian gravitational potential

$$
U \approx-\frac{G \mathcal{M}_{0}^{2}}{2 r_{s}}
$$

assuming an exponential disk profile of scale length $r_{s}$ and total baryonic mass $\mathcal{M}_{0}$. Equation (1) corrects a missing minus sign in Eq. (16b). The contribution to the Newtonian potential from the symmetron is negligible. However, additional potential energy results from the direct coupling between the baryons and the symmetron:

$$
E_{\varphi}=\int \mathrm{d} V \frac{\rho \varphi^{2}}{2 M^{2}} \approx \frac{\mathcal{M}_{0}}{2}\left(\frac{v}{M}\right)^{2} \int_{0}^{\infty} \mathrm{d} x x e^{-x}\left(\frac{\varphi}{v}\right)^{2}
$$

where $\mathrm{d} V$ is the three-volume element and $x \equiv r / r_{s}$. Equation (2) supersedes Eq. (17). The remaining integral, which we denote by the dimensionless parameter $\alpha$, is of order unity if the symmetron field does not vary significantly from its vacuum expectation value, i.e., $\varphi \sim v$, as is the case for the chosen value of $\mu=3 \times 10^{-39} \mathrm{GeV}$. We therefore find

$$
t=\frac{T}{\left|U+E_{\varphi}\right|} \approx\left|-\frac{f_{0}^{2}}{8}+\frac{\alpha}{8 g_{\dagger} r_{s}}\left(\frac{v}{M}\right)^{2}\right|^{-1},
$$

which replaces the incorrect expression in Eq. (18). For $f_{0} \approx 5$, stability of the galactic disk yields the following constraint on the ratio $v / M$ :

$$
\frac{v}{M} \gtrsim \frac{4 \times 10^{-3}}{\sqrt{\alpha}}
$$

This replaces the incorrect constraint on the mass $\mu$ in Eq. (19), and it is satisfied for $v / M=1 / 150$ for reasonable values of $\sqrt{\alpha} \gtrsim 3 / 5$. (We note that the parameter $\alpha$ depends implicitly on $\mu$.) The conclusions of the original article are therefore unaltered: the energy stored in the interaction between the symmetron field and the baryonic component is sufficient to stabilize the galactic disk.

[1] J. P. Ostriker and P. J. E. Peebles, Astrophys. J. 186, 467 (1973). 